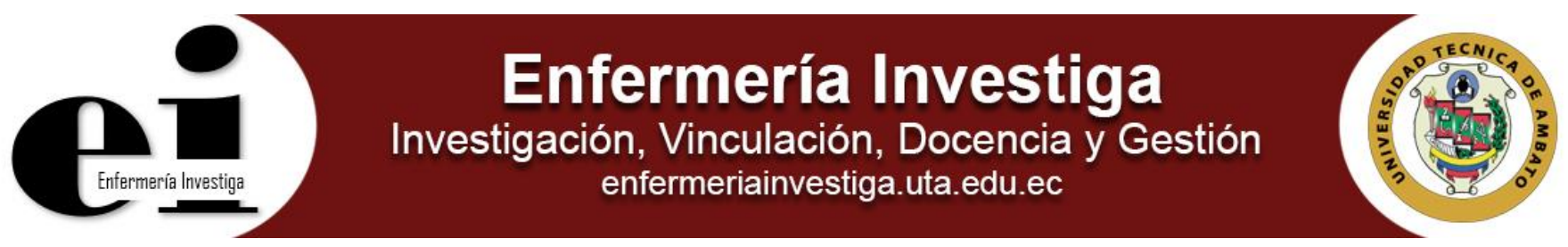

Artículo revisión

\title{
¿Qué afecta más al estudiante universitario, vivir solo o en una familia disfuncional?
}

\author{
Yesenia Acosta ${ }^{1}$, Gabriela Monroy², Rodrigo Alvear ${ }^{1}$, Susana Meneses ${ }^{1}$ \\ ${ }^{1}$ Universidad Técnica del Norte - Facultad Ciencias de la Salud - Ambato - Ecuador \\ ${ }^{2}$ Clínica Metropolitana - Ibarra - Ecuador
}

Acosta Y, Monroy G, Alvear R, Meneses S, ¿Qué afecta más al estudiante universitario, vivir solo o en una familia disfuncional? Enferm Inv. 2019; 4(2):3-7

2477-9172 / 2550-6692 Derechos Reservados (@) 2019 Universidad Técnica de Ambato, Carrera de Enfermería. Este es un artículo de acceso abierto distribuido bajo los términos de la Licencia Creative Commons, que permite uso ilimitado, distribución y reproducción en cualquier medio, siempre que la obra original es debidamente citada.

\section{Historia:}

Recibido: 15 octubre 2018

Revisado: 14 noviembre 2018

Aceptado: 18 marzo 2019

Palabras Claves: Familia disfuncional, salud mental, estudiante universitario.

\section{Keywords:}

Dysfunctional family, mental health, university student

\begin{abstract}
Resumen
Se conoce como trastorno mental al síndrome o a patrón de carácter psicológico que por lo general se asocia a un malestar o a una discapacidad. Es interesante destacar que una enfermedad mental es aquella que se produce a raíz de una alteración que repercute sobre los procedimientos afectivos y cognitivos del desarrollo, la cual se traduce en dificultades para razonar, alteraciones del comportamiento, impedimentos para comprender la realidad y para adaptarse a diversas situaciones.
\end{abstract}

\begin{abstract}
It is known as mental disorder to the syndrome or pattern of psychological character that is usually associated with a malaise or a disability. It is interesting to note that a mental illness is one that occurs as a result of an alteration that affects the affective and cognitive procedures of development, which translates into difficulties in reasoning, alterations in behavior, impediments to understanding reality and adapting to various situations.
\end{abstract}

Autor de correspondencia:

Susana Meneses. Universidad Técnica del Norte - Teléfono: +593 999443617, Ibarra, Ecuador. Email: msmeneses@utn.edu.ec 


\section{Introducción}

Se conoce como trastorno mental al síndrome o a patrón de carácter psicológico que por lo general se asocia a un malestar o a una discapacidad. Es interesante destacar que una enfermedad mental es aquella que se produce a raíz de una alteración que repercute sobre los procedimientos afectivos y cognitivos del desarrollo, la cual se traduce en dificultades para razonar, alteraciones del comportamiento, impedimentos para comprender la realidad y para adaptarse a diversas situaciones.

Se han realizado diversos estudios para evaluar la salud mental de los estudiantes universitarios en algunas partes del mundo.

En Malasia se realizó una revisión sistemática en el cual se arrojó entre los diversos resultados, que hay variables que influyen negativamente en la salud mental causando enfermedades como el estrés. De hecho, se determinó que los alumnos que cursaban la carrera de medicina tenían el porcentaje de estrés más alto comparado con las demás facultades. Las variables externas que influenciaron van desde la relación con padres y maestros, hasta temas relacionados con la situación financiera. (1)

Es importante darle la debida atención a la salud mental, es por ello que en otro meta análisis se investigó la efectividad de programas de prevención de salud mental en la educación superior, dando como resultado un mayor grado de efectividad, los programas con supervisión son más efectivos que los que carecen de ella para reducir significativamente la depresión, ansiedad, estrés y angustia psicológica general y para mejorar habilidades socioemocionales, auto percepción y comportamientos académicos. (2)

La depresión es uno de los trastornos mentales que más afectan a los estudiantes, tomando en cuenta que siempre se acompaña de más síntomas que incluso pueden afectar gravemente la salud no solo mental sino física. Los trastornos mentales comunes ocupan el segundo lugar, cuya sintomatología afecta el estado de ánimo, dando sensación de ansiedad y problemas alimenticios, aquí se excluye a los trastornos psicóticos.

Se conoce que los estudiantes más susceptibles a problemas de salud mental son aquellos de las carreras de medicina o relacionado a ciencias de la salud. (4) (5).

Se debe tomar en cuenta que muchos estudiantes llegan a la universidad ya con problemas de salud mental de base, es por eso que se debe hacer énfasis no solo en los procesos de aprendizaje sino en programas que ayuden a los estudiantes a conocerse a sí mismo e identificar sus afecciones, para así sobrellevar de una mejor manera su salud y que no interfiera con su plan de estudios. Todo este proceso debe ir de la mano del apoyo de autoridades. (6)

\section{Métodos}

Se realizó un estudio descriptivo transversal, cuya muestra fue de 309 estudiantes hombres y mujeres entre 18 a 26 años, de la Universidad Técnica del Norte, de ellos se seleccionó para el análisis aquellos que al momento de la entrevista vivían solos. Se analizó los resultados obtenidos con el SRQ-30 y el grado de percepción de disfuncionalidad familiar a través de cruce de variables con chi-cuadrado para determinar asociación entre vivir solos, disfunción familiar y el riesgo de trastornos mentales.

\section{Resultados}

Los resultados evidencian que, de los 309 estudiantes, el $60 \%$ tiene algún grado de disfunción familiar y el 56\% salieron positivos al test de SRQ-30 y el 14\% viven solos. Al cruce de variables se observa que la disfunción familiar se asocia más a la probabilidad de tener un trastorno mental que el vivir solos.

Esto nos indica que la mayor concentración de trastornos mentales medidos por la positivada del test SRQ-30, se encuentra en estudiantes con familias con algún grado de disfuncionalidad sea esta severa, moderada o leve (chi2=11,368a, gl=3, $p<0.01$ ) 


\section{Figura 1}

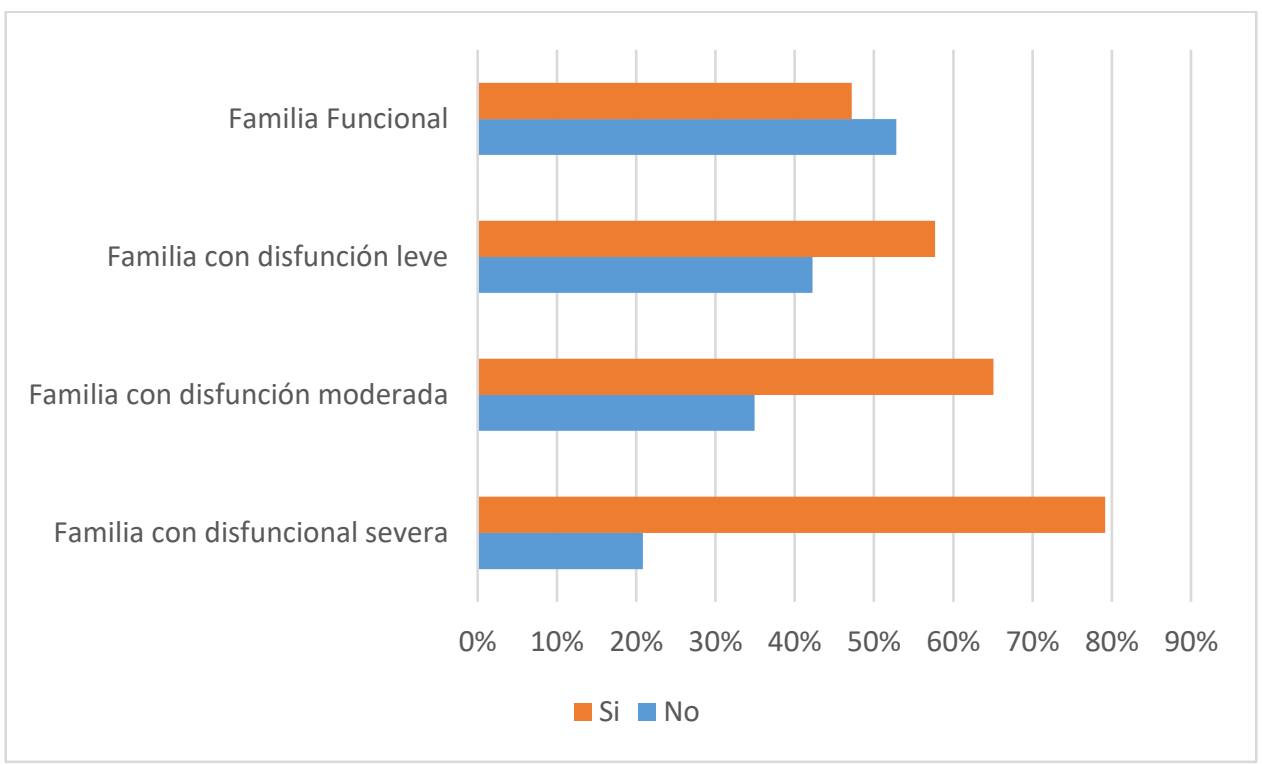

Por otro lado, al efectuar el cruce con los resultados del Test Apgar con el trastorno de Neurosis, encontramos que en los estudiantes que son parte de familias con disfunción severa son más propensos a presentar un cuadro de Neurosis (67\% presenta el trastorno); en familias con disfunción moderada un $44 \%$ resultó positivo, familias con disfunción leve $30 \%$ resultó positivo y familias funcionales $24 \%$ resultó positivo (chi2=20,915a, gl=3, $\mathrm{p}<0.001$ ).

\section{Figura 2}

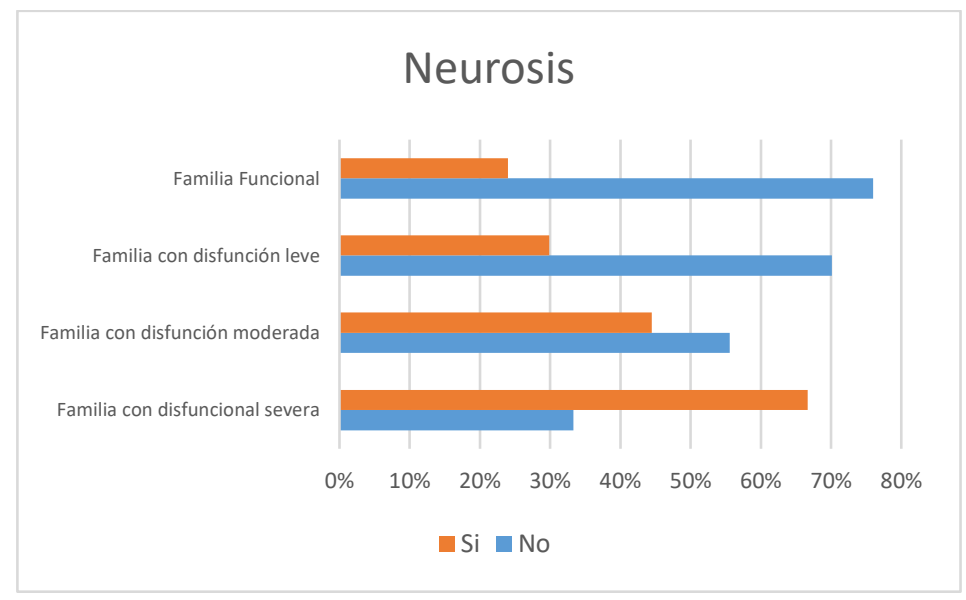

Analizando otro tipo de trastorno y efectuando un cruce similar, podemos determinar que los estudiantes con familias con disfunción severa tienden a presentar trastornos psicóticos (un $46 \%$ dio positivo a los resultados); en familias con disfunción moderada un $22 \%$ resultó positivo, familias con disfunción leve $29 \%$ resultó positivo y familias funcionales $18 \%$ resultó positivo leve (chi2=9,569a, $g l=3, p<0.025$ ) 


\section{Figura 3}

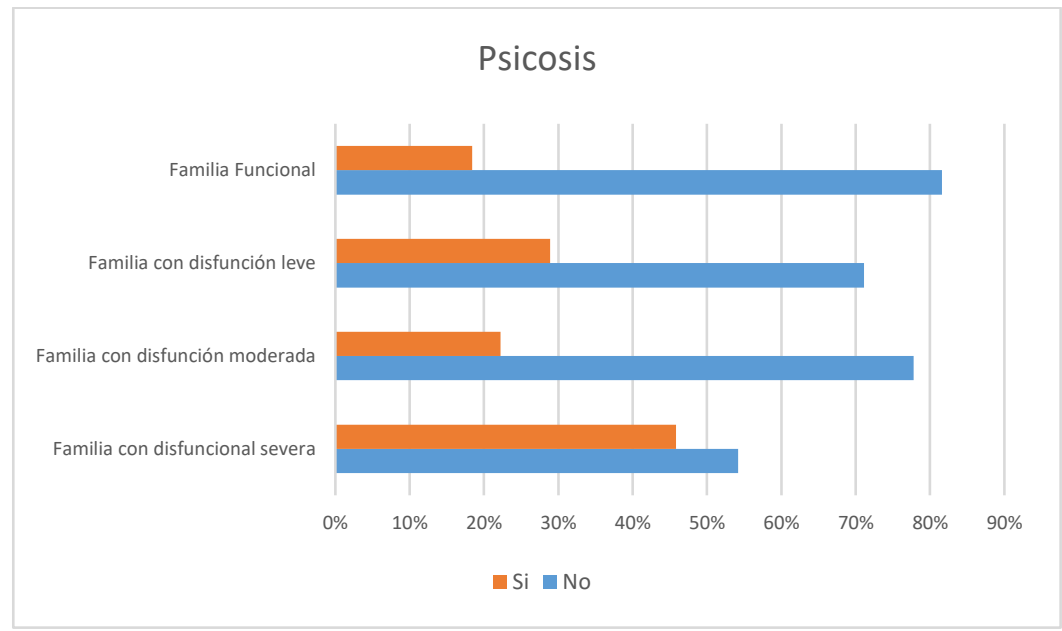

La epilepsia y el alcoholismo no presentaron variaciones significativas mostrando resultado parejo entre las distintas categorías de familias. De los 309 estudiantes encuestados, únicamente 42 viven solos (14\%), mientras que los 267 restantes (86\%) viven en familia. La asociación de esta variable no presenta ninguna significancia estadística.

\section{Discusión}

La variable que más se asocia, en los estudiantes, a riego de padecer trastornos mentales es la disfunción familiar muy por encima de vivir solos. La patología que más se asocia a disfunción familiar es la neurosis que engloba a patologías como la depresión y la ansiedad.

Estudios previos se enfocan en subgrupos como son los estudiantes de medicina y el riesgo que contraer enfermedades de este tipo, los cuales se determina que el estrés y la depresión son las patologías más comunes y que afectan las carreras de la salud en mayor intensidad que otras.

Esto se debe al nivel de presión y además a la carga psicológica que tienen que enfrentar. (1,3-5). Partiendo de este antecedente se realizaron más estudios no solo enfocados a estudiantes de aéreas de la salud, sino también a otras carreras, y así mismo se investigaron programas que ayuden a evitar trastornos mentales en estudiantes, llegando a la conclusión que los programas supervisados eran más efectivos que aquellos que no tenían supervisión. (2)

Conociendo esto, este estudio se enfocó en el tipo de funcionalidad familiar y el papel que juega este en el desarrollo de una buena salud mental. La familia debe trabajar la parte afectiva de todos sus miembros dando espacio a la comunicación, creando la predisposición para asistir y formar parte del proceso de aprendizaje y enseñanza para el bienestar de sus hijos en todas las etapas del ciclo vital.

Se realizó además otro estudio en donde se demostró que una persona que tiene una familia disfuncional es más propensa a tener un bajo rendimiento académico lo cual ayuda a desarrollar estrés, ansiedad y depresión, es por esto que la familia tiene un papel fundamental en la formación personal no solo académicamente hablando, sino en salud tanto mental como física. (7)

\section{Conclusiones}

Además, se puede concluir que un factor que colabora a la manifestación de los distintos trastornos analizados es el tipo de la familia a la que el individuo forma parte.

El estudio arrojo que si hay relación entre grado de familia disfuncional con presentación de trastornos mentales en los estudiantes universitarios; se debe realizar análisis de casos y prestar ayuda psicológica a las personas de este grupo susceptible.

Individuos que son parte de familias con un grado de disfunción severa, manifestaron en mayor proporción los trastornos de Neurosis, Psicosis, Epilepsia y Alcoholismo frente a las demás categorías, por lo que es importante el entorno familiar que rodea al individuo para evitar el desarrollo de estas patologías.

Los estudiantes que viven solos son menos susceptibles al desarrollo de trastornos mentales. Se recomienda realizar más estudios acerca del tipo de familia y salud mental. 


\section{Conflicto de intereses}

Ninguno declarado por los autores.

\section{Financiación}

Autofinanciado.

\section{Agradecimientos}

Ninguno declarado por los autores.

\section{Referencias}

1. Salam A, Yousuf R, Bakar SMA, Haque M. Stress among medical students in malaysia: A systematic review of literatures. Int Med J. $2013 ; 20(6): 649-55$.

2. Conley CS, Durlak JA, Kirsch AC. A Meta-analysis of Universal Mental Health Prevention Programs for Higher Education Students. Prev Sci. 1 de mayo de 2015;16(4):487-507.

3. Pacheco JP, Giacomin HT, Tam WW, Ribeiro TB, Arab C, Bezerra IM, et al. Mental health problems among medical students in Brazil: a systematic review and meta-analysis. Rev Bras Psiquiatr. 31 de agosto de 2017;39(4):369-78.

4. Hope V, Henderson M. Medical student depression, anxiety and distress outside North America: a systematic review. Med Educ. 1 de octubre de 2014;48(10):963-79.

5. Cuttilan AN, Sayampanathan AA, Ho RC-M. Mental health issues amongst medical students in Asia: a systematic review [2000-2015]. Ann TransI Med [Internet]. febrero de 2016 [citado 8 de abril de 2018];4(4). Disponible en: https://www.ncbi.nlm.nih.gov/pmc/articles/PMC4779785/

6. Ennals P, Fossey E, Howie L. Postsecondary study and mental ill-health: a meta-synthesis of qualitative research exploring students' lived experiences. J Ment Health. 4 de marzo de 2015;24(2):111-9.

7. López Mero P, Barreto Pico A, Rodríguez E, Rigoberto E, Bello del S, Alberto MW. Bajo rendimiento académico en estudiantes y disfuncionalidad familiar. MEDISAN. septiembre de 2015;19(9):1163-6. 